\title{
Courant Institute of
}

Mathematical Sciences

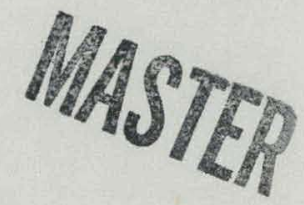

Magneto-Fluid Dynamics Division

\section{Hall Current Effects on Tearing Modes in Rotating Reverse Field Plasmas}

†Jay Kappraff, William Grossmann, Michael Kress

U. S. Department of Energy

and

Air Force Office of Scientific Research Report

Plasma Physics

March 1980

New York University

†New Jersey Institute of Technology 


\section{DISCLAIMER}

This report was prepared as an account of work sponsored by an agency of the United States Government. Neither the United States Government nor any agency Thereof, nor any of their employees, makes any warranty, express or implied, or assumes any legal liability or responsibility for the accuracy, completeness, or usefulness of any information, apparatus, product, or process disclosed, or represents that its use would not infringe privately owned rights. Reference herein to any specific commercial product, process, or service by trade name, trademark, manufacturer, or otherwise does not necessarily constitute or imply its endorsement, recommendation, or favoring by the United States Government or any agency thereof. The views and opinions of authors expressed herein do not necessarily state or reflect those of the United States Government or any agency thereof. 


\section{DISCLAIMER}

Portions of this document may be illegible in electronic image products. Images are produced from the best available original document. 
New York University

Courant Institute of Mathematical Sciences

Magneto-Fluid Dynamics Division

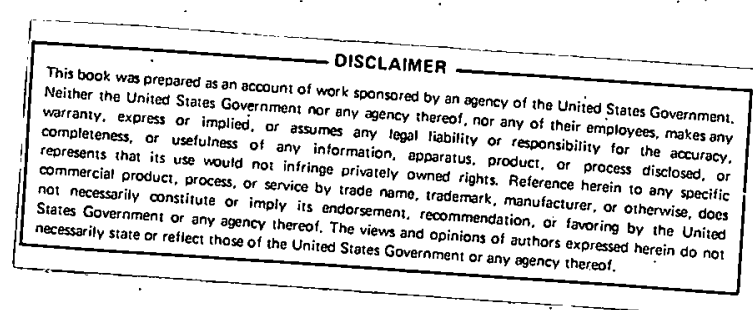

Hall Current Effects on Tearing Modes

in

Rotating Reverse Field Plasmas

Jay Kappraff ${ }^{\dagger}$, William Grossmann, Michael Kress March 1980

U. S. Department of Energy

Contract No. DE-ACO2-76ERO3077

and

Air Force Office of Scientific Research

Contract No. $76-2881$

$\dagger$ - New Jersey Institute of Technology.

UNCLASSIFIED 


\section{ABSTRACT}

It has been experimentally observed for some time that certain tearing modes in plasmas may be suppressed if the plasma rotates in a preferred direction. In this paper we treat the $m=0$, finitc wavclongth tcaring mode in cylindrical geometry for a reversed field plasma equilibrium and show that by generalizing ohm's law to. include Hall current terms, we are able to explain this effect of rotation on tearing modes. Our results agree qualitatively with earlier analysis and numerical simulations of A. Kaleck. We also show that our results are sensitive to the position of the outer conducting wall, and for wall positions sufficiently close to the plasma vacuum interface, tearing. modes may be quenched. when the rotation reaches a critical value. These results follow from a boundary layer analysis and numerical integration of the boundary layer equations. 


\section{INTRODUCTION}

The purpose of the present work is to re-condider a well-known resistive MHD instability by considering the effect of a more general form of ohm's Law. Specifically, we treat the m=o, finite wavelength tearing mode in cylindrical geometry for a reversed field plasma equilibrium typical of those which are produced in reverse-bias theta pinch experiments. (BODIN, 1963; KALEK et al., 1968; EBERHAGEN and GROSSMANN, 1971; ES'KOV et al., 1978; LINFORD et al., 1978). The motivation for the present study stems from early experiments which showed a curious and unexplained absence of an expected tearing mode (EBERHAGEN and GROSSMANN, 1971). Reverse bias theta pinch experiments generally produce a closed field configuration with reverse trapped magnetic flux; this occurs due to magnetic line reconnection at the coid ends of the pinch coil where the resistivity is high. The formation of the closed region is also associated with a strong axial contraction as the plasma attempts to find an equilibrium. The result is an elongated (length to width ratio > 10) reversedfield plasma with a bounding closed separatrix, which according to the simplest classical picture should be further susceptable to tearing modes, $\mathrm{m}=0$ and $\mathrm{k}_{\mathrm{z}} \neq 0$, which would tend to break the column into a number of cells each having reversed trapped flux. The number and size of the cells as predicted by the simple linear theory varies from experiment to experiment since the linear dispersion relation depends sensitively on plasma profiles. The classical tearing mode theory due to FURTH, KILLEEN and ROSENBLUTH (1963) carried out originally in slab geometry yields, for cylindrical geometry (KALEK, 1972) 
the prediction that most of the experimentally produced reversed field theta pinch plasmas should break up into reversed field cells. Experimental results of EBERHAGEN and GROSSPANN (1971) and more recently by LINFORD (1978) show that the expected tearing mode does not occur and the plasmas in question exist for some tens of linear growth times. Instead of the expected tearing a pronounced rotation of the plasma column is observed; this rotation increases with time until the rotational frequency reaches a critical value at which time the plasma goes catastrophically unstable to an $\mathrm{m}=2$ mode. In an attempt to explain the absence of the tearing modes KALEK (1972) simulated the plasma behaviour with a linearized initial value MHD code assuming the plasma column to be infinitely long with no longitudinal spatial dependence. Kalek's model originally contained the resistive MHD equations of motion linearized about a rigidly rotating plasma equilibrium; results from the numerical code showed that the inclusion of rotation predicted even larger growth rates than for the static equilibrium case. The addition of the Hall effect into the Ohm's Law yielded results which were not inconsistent with the experimental results, namely the growth rates as calculated were at least an order of magnitude lower than what the FKR theory would predict; this statement requires qualification in that the relevant part of the FKR cylindrical tearing mode dispersion relation is distorted in a manner to suppress the growth rate. The long wavelength $(k a \leq 1)$ part of the dispersion relation is affected and a preferred direction of rotation is indiçated for a stabilizing influence. The direction of the ion 
diamagnetic drift velocity is preferred for stability and rotation in the opposite direction results in enhanced instability. Ralek's numerical results lend strong support to a beneficial interplay between the effects of rotation and Hall current in the suppression of tearing mode growth rates. The calculation described in the present paper attempts to analytically investigate the $m=0 ; k_{z} \neq 0$ tearing mode in reversed field plasmas and attempts to explain the numerical results of KALEK. Our results are consistent, with Kalek's and we find a stabilizing influence of rotation combined with Hall effect; however, we find that the results are very sensitive to profiles, wall position and plasma parameters such as density, temperature and rotation frequency: The specific calculation presented in the present paper deals with plasma profiles which are representative of those which are produced in reversed field theta pinch experi-. ments.

It should be mentioned that the use of Ohm's Law with a Hall term has also been used to model the effect of finite particle effects in treating tearing modes since the Hall term is associated with a coefficient which is proportional to the ion gyration frequency (COHEN et al., 1977). In such a case overstable modes can be found. In the problem treated here the $\mathrm{m}=0$ mode does not admit overstable solutions; this is not explicitly pointed out in the text but follows from simple energy arguments.

The paper is organized into 9 sections. The governing equations are presented in section 2. The governing equations are non-dimensionalized in Section 2. Solutions of the governing 
$-4-$

equations in the resistive boundary layer are discussed in Sectron 3. Solutions outside the resistive layer are discussed in Section 4 and matching across the boundary layer is discussed in section 5. The tearing mode ordering with a small parameter is discussed in Section 6. Sections 7 and 8 discuss the compotation of growth rates for specific equilibrial. A final secLion presents the conclusions of the computations discussed here. 
2. THE MODEL:

We consider a straight cylindrical plasma column whose equilibrium profiles are considered to have variation only in the radial direction. The plasma is considered incompressible and has a density profile, $\rho_{0}(r)$, that extends to a plasmavacuum interface where the density is zero. The equilibrium magnetic field, $\underline{B}_{0}^{\prime}(r)$ has both longitudinal and azimuthal components. Finally, the column is subjected to a rotation with rotational frequency $\Omega(\mathrm{r})$.

2.1 Basic equations

To study this system we employ the following mathemical model:

$$
\begin{aligned}
& \frac{\partial \rho}{\partial t}+\nabla \cdot(\rho \underline{v})=0
\end{aligned}
$$

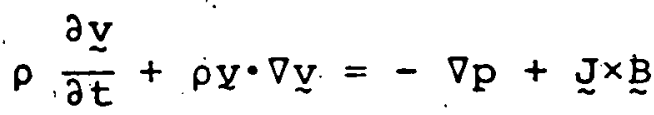

$$
\begin{aligned}
& \frac{\partial \underline{\mathrm{B}}}{\partial \mathrm{t}}+\nabla \times \underset{\sim}{\mathrm{E}}=0 \\
& \underline{J}=\frac{1}{\mu_{0}} \nabla \times \underset{\sim}{B} \\
& \underline{E}=-\underline{\underline{V}} \times \underline{\underline{B}}+n \underset{\sim}{J}+\frac{\varepsilon}{\dot{\varepsilon}} \underset{\sim}{J} \times \underline{B} \\
& \nabla \cdot \underline{v}=0 \\
& \nabla \cdot \underline{B}=0
\end{aligned}
$$


where $\mathrm{V}$ is the plasmia velocity, $\underset{\sim}{\mathrm{B}}$ the magnetic field, $p$ the plasma pressure, J the current density, E the electric field and $n, \varepsilon$ are resistivity and Hall current parameters respectively which are taken to be constant. The mathematical model specified by Equations (1) - (7) differs from Ideal MHD only through Ohm's Law which, includes the effect of finite resistivity and Llè Hall effecl. Our dill in this report is to study the time behavior of small perturbations about an equilibrium specified by $\underset{\sim 0}{\mathrm{~V}}(r),{\underset{\sim}{\mathrm{B}}}_{\mathrm{O}}(r), \rho_{\mathrm{o}}(r)$ and $\mathrm{p}_{\mathrm{O}}(r)$. The perturbed velocity field, magnetic field, density, pressure, current density and electric field are represented by $\underset{\sim}{\mathrm{u}}, \underset{\sim}{\mathrm{b}}, \delta \rho, \delta p, \underset{\sim}{j}$ and $\delta \underset{\sim}{\mathrm{E}}$ respectively.

The linearized version of equaliums of (1) - (7) are:

$$
\begin{aligned}
& \frac{\partial}{\partial t} \delta \rho+\nabla \cdot\left(\delta \rho \underline{-}_{0}\right)+\nabla \cdot\left(\rho_{0} \underline{u}\right)=0 \\
& \frac{\partial}{\partial t} \rho_{0} \underline{u}=-\dot{\nabla}_{p} \star+\underset{\sim}{j} \times \sim_{0}+\underset{\sim}{J_{0}} \times \underset{b}{\underline{b}}-\rho_{0} \underset{v}{\underline{u}} \nabla \underline{\sim}_{0} \\
& -\rho_{0} \underline{v}_{0} \cdot \nabla \underline{u}-\delta \rho \underline{v}_{0} \cdot \nabla \underline{v}_{0}+\frac{1}{\mu_{0}} \nabla(\underline{B} \cdot \underline{b}) \\
& \frac{\partial}{\partial t} \underset{b}{b}=-\nabla \times \delta \underline{E} \\
& j=\frac{1}{\mu_{0}} \nabla \times \underline{b}
\end{aligned}
$$

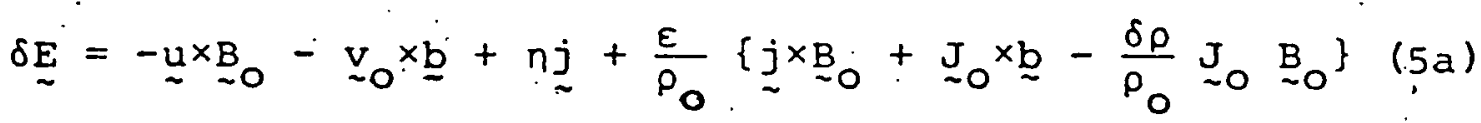




$$
\begin{aligned}
& \nabla \cdot \underline{u}=0 \\
& \nabla \cdot \underline{w}=0
\end{aligned}
$$

The starting set of equations for the computation can be obtained from system (la) - (7a) by Fourier transforming the perturbed quantities in the ignorable $\theta, z$ directions and time, $t$. As mentioned, we consider only the mode $m=0, k \neq 0$, where $m$ is the azimuthal wave number and $k$ is the axial wave number. Thus,

$$
\begin{aligned}
& \underline{b}=\underset{\tilde{b}}{(}(r) \exp (i[\omega t-k z]) \\
& \underline{u}=\underset{\sim}{\tilde{u}}(r) \exp (i[\omega t-k z])
\end{aligned}
$$

etc.

For ease of notation we will omit superscripts. from the perturher transformed variables.

After transforming equations (1a) - (7a) and rearranging we arrive at the following sixth order system of ordinary differential equations:

$$
\begin{aligned}
& \frac{d}{d r}\left(\rho_{0} \omega^{2} \frac{1}{r} \frac{d\left(r \xi_{r}\right)}{d r}\right)-i \frac{f}{\mu_{0}} \frac{d}{d r}\left(\frac{d}{r} \frac{d\left(r b_{r}\right)}{d r}\right)=-k^{2} \rho_{0}\left(r \Omega^{2} \frac{d \rho_{0}}{\frac{d r}{\rho_{0}}}\right. \\
& \left.-\omega^{2}+4 \Omega^{2}+2 r \Omega \frac{d \Omega}{d r}\right) \xi_{r}+i\left(\frac{1}{\mu_{0}}\left[-\frac{d^{2} f}{d r^{2}}+\frac{1}{r} \frac{d f}{d r}-k^{2} f^{2}\right]\right. \\
& \left.-\frac{2 k^{2} \Omega}{\omega} J_{O, z}\right) b_{r}-\left(\frac{2 k^{2} f}{\omega n H_{0}} \Omega+\frac{2 B_{\theta}}{r} k^{2}\right) b_{\theta}
\end{aligned}
$$


$-8-$

$$
\begin{aligned}
& \frac{n}{\mu_{0}} \frac{d}{d r} \cdot\left(\frac{1}{r} \cdot \frac{d}{d r}\left(r b_{r}\right)\right)=-f \omega \xi_{r}+\frac{\varepsilon f k}{\mu_{0} \rho_{0}} b_{\theta} \\
& \therefore \quad+\left(i \omega+\frac{k^{2} n}{\mu_{0}}+\frac{i \varepsilon}{\rho_{0}} k J_{0, z}\right) b_{r} \\
& \frac{\varepsilon f}{\mu_{0} \rho_{0}} \frac{d}{d r}\left(\frac{1}{r} \frac{d}{d r}\left(r b_{r}\right)\right)-\frac{n k}{\mu_{0}} \frac{d}{d r}\left(\frac{1}{r} \frac{d}{d r}\left(r b_{\theta}\right)\right) \\
& =-k\left(i \omega+\frac{n k^{2}}{\mu_{0}}-\frac{i f^{2}}{\omega \mu_{0} \rho_{0}}+\frac{i \varepsilon k_{0}}{\mu_{0}}\left[\frac{2 B_{\theta}}{r \rho_{0}}+\frac{B_{\theta}}{\rho_{0}} \frac{d \rho_{\theta}}{d r}\right]\right) \cdot b_{\theta} \\
& +i\left(-\frac{\varepsilon}{\rho_{0} 2}, \frac{d \rho_{o}}{d r}\left[\frac{d}{d r} \frac{f^{2}}{2 \mu_{0}}+k^{2} B_{0, \theta} J_{o, z}\right]\right. \\
& \left.-k \omega\left[J_{0, z}-\frac{2 B_{0, \theta}}{\mu_{0}^{r}}\right]+2 k f \Omega+k r f \frac{d \Omega}{d r}\right) \xi_{r} \\
& i\left(\frac{\dot{c}}{\mu_{0}}\left[r \cdot \frac{d}{d r}\left(\frac{1}{r \rho_{0}} \frac{d f}{d r}\right)+\frac{k^{2} f}{\rho_{0}}\right]+k r \frac{d \Omega}{d r}-\frac{k f f}{\omega \rho_{0}} J_{0, z}\right) b_{r}
\end{aligned}
$$

where $f=\mathrm{kB}_{\mathrm{O}, z}$. Furthermore, we have considered the equilib-. rim velocity field to be a rotation, ${\underset{\sim}{0}}_{0}=r \Omega(r) \hat{\theta}$. where $\hat{\theta}$ is a unit vector in the azimuthal direction. 
2.2 The equilibrium

We consider an equilibrium magnetic field profile with vanishing. $B_{0, z}$ on a cylindrical surface, $r=r_{0}$ in the plasma, i.e., a reversed field, while the component, $B_{O^{\prime} \theta}$ of the field is finite on $r=r_{0}$.

since the plasma is incompressible, we may impose an arbitrary density profile. Finally, as mentioned, the equilibrium flow is taken to be a rotation with variation in the radial direction.

The above equilibrium may be summarized as:

$$
\begin{aligned}
& \mathrm{B}_{\mathrm{O}}(r)=\mathrm{B}_{0, \theta} \hat{\theta}+\mathrm{B}_{0, z} \hat{z}^{\prime} \mathrm{B}_{0, z}\left(\mathrm{r}_{0}\right)=0 \\
& \underline{v}_{0}(r)=r \Omega(x) \hat{\theta} \text {. } \\
& p_{0}(x) \text { arbitrary. }
\end{aligned}
$$

2.3 Dimensionalization

We dimensionalize with respect to the equilibrium values at the neutral surface $r=r_{0}$ as follows:

$$
\bar{\eta}=\frac{\eta}{\omega_{0} a^{2} \mu_{0}}, \bar{\varepsilon}=\frac{\varepsilon}{\rho_{0} \omega_{0} \mu_{0}} \cdot \frac{d f}{d r}\left(r_{0}\right)
$$


$-10-$

$$
\begin{aligned}
& \bar{\rho}_{0}=\frac{\rho_{0}(r)}{\rho_{0}\left(r_{0}\right)}, \bar{f}=\frac{f}{\frac{d f\left(r_{0}\right)}{d r}}, \frac{d \bar{f}}{d r}=\frac{\frac{d f}{d r}}{\frac{d f}{d r}\left(r_{0}\right)} \\
& \overline{\frac{d^{2} f}{d r^{2}}}\left(r_{0}\right)=a \frac{\frac{d^{2} f}{d r^{2}}\left(r_{0}\right)}{\frac{d f}{d r}\left(r_{0}\right)}=\alpha_{1}, \tau=\frac{r-r_{0}}{a}, \bar{r}=\frac{r}{a} \\
& D_{s}=-\frac{a}{\rho_{0}} \frac{d \rho_{0}}{d r}, \bar{B}_{\theta}\left(r_{0}\right) \cdots \frac{B_{0, \theta}}{a^{2} \frac{d f}{d r}\left(r_{0}\right)} \\
& \bar{J}_{z}=\frac{J_{0, z}}{a \frac{d f}{d r}\left(r_{0}\right)}, \quad \bar{\gamma}=\frac{i \omega}{\omega_{0}}, \bar{\xi}=a \xi_{r} \\
& \bar{\Omega}=\frac{2 \Omega \bar{k}}{\omega_{0}}, \bar{k}=k a, \frac{d \bar{\Omega}}{d r}=\frac{2 a \bar{k}}{\omega_{o}} \frac{d \Omega}{d r}
\end{aligned}
$$

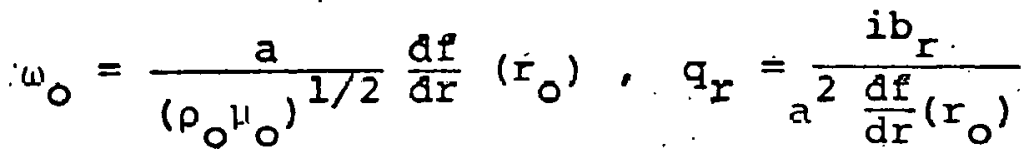$$
q_{\theta}=\frac{1 \bar{k} b_{\theta}}{a^{2 \frac{d f}{d r}\left(r_{0}\right)}}
$$

With these dimensionalizations:

$$
\bar{f}(\tau)=\tau+\frac{\alpha_{1}}{2} \tau^{2}+\ldots
$$

and,

$$
\bar{J}_{0,2}=\alpha_{2} \tau+\ldots
$$


We rewrite equations. (8) - (10) in dimensionless form for a layer centered about $r_{0}$ that is sufficiently small to neglect variations in the equilibrium quantities. This layer will be referred to as the boundary. layer, and the equations governing the behavior of the resistive layer will be known as the resistive boundary layer equations or RLE. To leading order in $\tau$ and omitting the superscripts on the dimensionless quantities, the RLE are:

$\gamma^{2} \frac{d^{2} \xi r}{d \tau^{2}}=-\tau \frac{d^{2} q_{r}}{d r^{2}}+\left(\Omega^{2}\left[1-\frac{r_{O} D_{s}}{4}\right]+\frac{r_{0}}{2} \Omega\left(\frac{d \Omega}{d r}\right]+k^{2} \gamma^{2}\right) \xi_{r}$

$$
-\left(\frac{1}{r_{0}}-k^{2} \tau-\alpha_{1}-\frac{i \Omega k \alpha}{\gamma}\right) q_{r}+\left(\frac{\Omega \tau}{\gamma}-\frac{2 i B_{\theta}}{r_{0}}\right) q_{\theta}
$$

$$
\begin{aligned}
& \eta \frac{d^{2} q_{r}}{d \tau^{2}}=-\gamma \tau \xi_{r}+\varepsilon \tau q_{\theta}+\left(\gamma+k^{2} \eta+i \varepsilon k \dot{\alpha}_{2} \tau\right) q_{r} \\
& \eta \frac{d^{2} q_{\theta}}{d \tau^{2}}-\varepsilon \tau \frac{d^{2} q_{r}}{d \tau^{2}}=\left(\gamma+\eta k^{2}+\frac{\tau^{2}}{\gamma}+i \varepsilon k B_{\theta}\left[\frac{2}{r}-D_{S}\right]\right) q_{\theta}
\end{aligned}
$$

$$
\begin{gathered}
+\left(D_{s}\left[1+k^{2} \alpha_{2}{ }_{\theta}\right]+\Omega \tau\left[1+\frac{r_{0}}{2 \Omega} \frac{d \Omega}{d r}\right]+\gamma\left[\alpha_{2} \tau-\frac{2 i k B}{r_{0}}\right]\right) \xi_{r} \\
-\left(\varepsilon\left[\alpha_{1}-\frac{1}{r_{0}}+D_{s}+k^{2} \tau\right]-\frac{i k \alpha_{2}}{r} \tau^{2}+\frac{r_{o}}{2} \frac{d \Omega}{d r}\right) q_{r} \quad(10 a)
\end{gathered}
$$




\section{ANALYSIS OF THE RLE}

\subsection{Scaled equations}

We wish to study the interesting behavior governed by the RLE in the limit of small resistivity and Hall parameter $\eta$ and $\varepsilon$. Following earlier work (COPPI, et al., 1965) we adopt the following scaling based on a resistive layer thickness of the order of the skin depth. with respect to the diffusion time scale, i.e., $\gamma \tau^{2} \sim n$ where $\tau-\gamma \cdot$.

$$
\begin{gathered}
\gamma=\phi S, \tau=\phi x, \eta=\phi \bar{n}, \Omega=0(1) . \\
\varepsilon=\phi \bar{\varepsilon} \\
\xi_{r}=\xi+\ldots, q_{r}=\phi \psi+\ldots, . \\
q_{\theta}=\zeta+\cdots
\end{gathered}
$$

Also denote:

$$
P=\frac{r_{0}}{2 \Omega} \frac{d \Omega}{d r}, \delta=r_{0}^{2 B_{\theta}}
$$

where the superscripted variables are considered to be $0(1)$ quantities while $\phi$ is a small scale factor. The scaling of the Hall parameter and angular velocity were chosen so that terms with $\varepsilon$ and $\Omega$ would neither dominate nor disappear from the scaled equations in the lowest order. 
Finally, we again omit the superscripts in writing the scaled equations:

$$
\begin{gathered}
s^{2} \frac{d^{2} \xi}{d x^{2}}+x \frac{d^{2} \psi}{d x^{2}}=\Omega^{2}\left(1-\frac{r_{0}}{4} D_{s}+P\right) \xi+\left(\frac{\Omega x}{s}-i \delta\right) \zeta \\
n \frac{d^{2} \psi}{d x^{2}}=-s \times \xi+\varepsilon^{x} \zeta+s \psi \\
n \frac{d^{2} \zeta}{d x^{2}}-\varepsilon \frac{d^{2} \psi}{d x^{2}}=\left(s+\frac{x^{2}}{s}+i \varepsilon \delta\left[1-\frac{r_{0} D_{S}}{2}\right]\right) \zeta \\
+(x \Omega[1+P]-i \delta S) \xi-P \Omega \psi
\end{gathered}
$$

Making use of (12), equations (11) and (13) can be rewritten:

$$
\begin{aligned}
s^{2} \frac{d^{2} \xi}{d x^{2}}= & \left(\frac{x^{2} s}{n}+\Omega^{2}\left[1-\frac{r_{0} D}{4}+P\right]\right) \xi+\left(\frac{\Omega x}{s}-\frac{\varepsilon x^{2}}{n}\right. \\
& -i s) \zeta-\frac{x S}{n} \psi
\end{aligned}
$$

$\eta \frac{d^{2} \zeta}{d x^{2}}=\left(s\left[1+\frac{x^{2}}{s}\right]+\frac{\varepsilon^{2}}{\eta} x^{2}+i \varepsilon \delta\left[1-\frac{I_{0}}{2} D_{S}\right]\right) \zeta$

$$
+\left(x \Omega[1+P]-i \delta S-\frac{\varepsilon x^{2}}{n} S\right) \xi+\left(\frac{\varepsilon x S}{n}-P \Omega\right) \psi .
$$

Equations (11) - (13) show that in the presence of azimuthal components, $\mathrm{B}_{\theta}{ }$, of the equilibrium magnetic field, overstable modes are possible due to the imaginary terms 
in the equations. For this analysis we suppress these terms by considering only long wavelength modes with sufficiently. small $k$ which results in the elimination of $\delta$ and $\alpha_{2}$ from equations (11) - (13) to leading order. 
3.2 Asymptotic behavior of RLE

The'system (11a), (12), (13a) constitutes a sixth order homogeneous linear differential equation with an irregular singularity at $x=\infty$. The system may be rewritten in a standard form for irregular perturbation analysis (WASOW, 1965).

$$
\frac{d \underline{u}}{d x}=x \underset{z}{A}(x) \underset{\sim}{u}
$$

which has a set of solutions of the form:

$$
\underline{u}=\underline{z} \cdot e^{\stackrel{\lambda}{\approx} \frac{x^{2}}{2}+\ldots} x_{x}^{\stackrel{D}{z}} \underset{z}{\hat{z}}(x)
$$

where $\underset{\sim}{c}$ is a constant matrix and $\underset{\sim}{\lambda}, \underset{\sim}{D}$ are diagonal matrices.

The elements of $\underset{\sim}{\lambda}$ determine the exponential nature of the asymptotic solution to the inner equations. These elements, $\lambda_{i}$, are the eigenvalues of the matrix,

$$
\stackrel{A}{\sim}(\infty)=\left|\begin{array}{cccccc}
0 & 0 & 0 & 1 & 0 & 0 \\
0 & 0 & 0 & 0 & 1 & 0 \\
0 & 0 & 0 & 0 & 0 & 1 \\
\frac{1}{n Q}-\frac{\varepsilon}{n Q^{2}} & 0 & 0 & 0 & 0 \\
\frac{\varepsilon Q}{n}\left(\frac{1}{n Q}+\frac{\varepsilon^{2}}{n}\right) & 0 & 0 & 0 & 0 \\
0 & 0 & 0 & 0 & 0 & 0
\end{array}\right|
$$


Thus,

$$
\begin{aligned}
& \lambda_{1,2}=0,0 \\
& \lambda_{3,4}=\frac{1}{(n s)^{1 / 2}}\left(\frac{r}{2} \pm\left(1+\frac{r^{2}}{4}\right)^{1 / 2}\right) \\
& \lambda_{5,6}=-\frac{1}{(n s)^{1 / 2}}\left(\frac{r}{2} \pm\left(1+\frac{r^{2}}{4}\right)^{1 / 2}\right) .
\end{aligned}
$$

where $r^{2}=\frac{\varepsilon^{2} s}{n}$.

At this point it is not clear whether $\lambda_{k}$; $k:=3,4,5,6$ have pure or oscillating spatial exponential growth or decay, or'whether they have pure oscillatory behavior as $x \rightarrow \infty$. In fact, we can show that only if,

$$
S=\frac{i}{\left(\alpha^{2}+1\right) \varepsilon^{2}}\left(-1 \pm \frac{i}{\alpha}\right)
$$

for $\alpha$ an arbitrary real number, can $\lambda_{k}$ be pure imaginary in which case

$$
\lambda_{k}= \pm \frac{\varepsilon}{\eta} i
$$

Thus, all these solutions either decay or grow exponentially as $x \rightarrow \infty$ in the class of solutions with positive growth rates, i.e., Re $S>0$,

Finally we consider the two algebraic equations corresponding to $\lambda_{1}$ and $\lambda_{2}$. To determine the asymptotic behavior of these solutions as $x \rightarrow \infty$, we consider the 
dominant terms from equations (11a) and (13a), as $x \rightarrow \infty$ :

$$
\begin{gathered}
\frac{s \xi}{n}-\frac{\varepsilon \zeta}{n} \simeq \frac{s \psi}{x n} \\
\left(\frac{\Omega}{x}-\frac{\varepsilon S}{n}\right) \xi+\left(\frac{1}{S}+\frac{\varepsilon}{n}\right) \zeta=-\frac{\varepsilon S}{x \eta} \psi
\end{gathered}
$$

Solving to leading order in $x$ for $\xi$ and $\gamma$ yields:

$$
\begin{aligned}
& \xi \simeq \frac{\psi}{x} \\
& \zeta=-\frac{5 \Omega}{x^{2}} \psi
\end{aligned}
$$

Thus,

$$
\zeta \simeq-\frac{s \Omega}{x} \xi \cdot
$$

Also from (12),

$$
\frac{\mathrm{d}^{2} \psi}{d \mathrm{x}^{2}}=\frac{\mathrm{d}^{2}(\mathrm{x} \xi)}{\mathrm{dx^{2 }}}-\varepsilon \frac{\mathrm{d}^{2}}{d \mathrm{x}^{2}}\left(\frac{\mathrm{x} \zeta}{\mathrm{s}}\right)
$$

Replacing (14) and (15), in (11) and noting that

$$
x \frac{d^{2}}{d x^{2}}(x \xi)=\frac{d}{d x}\left(x^{2} \frac{d \xi}{d x}\right)
$$


$-18-$

yields, to leading order as $x \rightarrow \infty$,

$$
\frac{\mathrm{d}}{\mathrm{dx}}\left(\mathrm{x}^{2} \frac{\mathrm{d} \xi}{\mathrm{dx}}\right)=-\Omega^{2}\left(\frac{\mathrm{r}_{\mathrm{o}}}{4} \mathrm{D}_{\mathrm{S}}-\text { D }\right) \xi
$$

which has the solutions,

$$
\xi \sim x^{h}, x^{-1-h}
$$

where $h=-\frac{1}{2}+\frac{1}{2}\left(1-\Omega^{2}\left(\frac{{ }_{0}^{D} S}{4}-P\right)\right)^{1 / 2}$. In order to arrive at (16) we made use of the fact that in view of (14), $\gamma \sim x^{h-1}, x^{-2-h}$

In summary, the asymptotic solutions to system

(11)- (13) take the form, as $x \rightarrow \infty$,

$$
\begin{gathered}
\xi \sim \exp \left\{\frac{1}{(n s) 1 / 2}\left(\frac{r}{2} \pm\left(1, \frac{r^{2}}{4}\right)^{1 / 2}\right) \frac{x^{2}}{2}\right\} \\
\sim \exp \left\{\frac{-1}{(n s)^{1 / 2}}\left(\frac{r}{2} \pm\left(1+\frac{r^{2}}{4}\right)^{1 / 2}\right) \frac{x^{2}}{2}\right\} \\
\sim x^{n}, x^{-n-1}
\end{gathered}
$$

where $r=\frac{\varepsilon^{2} S}{n}, h=-\frac{1}{2}+\frac{1}{2}\left(1-\Omega^{2}\left(\frac{{ }^{\circ}}{4} D_{S}-P\right)\right)^{1 / 2}$. 


\section{SOLUTIONS OUTSIDE THE RESISTIVE LAYER}

The behavior of the plasma outside a narrow region surrounding the neutral. surface is governed to leading order by ideal MHD, i.e., $\varepsilon$ and $n$ can be taken to be zero. In terms of the same dimensionalizations as the resistive layer equations, the outer equations reduce, near marginal stability, i.e. $\gamma \approx 0$, to the following second order differential equation:

$$
\frac{d}{d r}\left(\frac{f^{2}}{r} \frac{d}{d r}\left(r \xi_{r}\right)\right)-f^{2} k^{2} \xi_{r}=-\frac{\rho_{0}}{4} r \Omega^{2}\left(D_{S}-\frac{2}{\Omega} \cdot \frac{d \Omega}{d r}\right) \xi_{r}
$$

Near $r_{0}$ equations (I1)-(13) reduce asymptotically to:

$$
\frac{d}{d \tau}\left(\tau^{2} \frac{d \xi_{r}}{d \tau}\right)=-\Omega^{2}\left(\frac{r_{0} D_{S}}{4}-P\right) \xi_{r}
$$

which has solutions,

$$
\xi=\tau^{h}, \tau^{-1-h}
$$

where $\mathrm{h}=-\frac{1}{2}+\frac{1}{2}\left(1-\Omega^{2}\left({ }^{r} D^{D} S-P\right)\right)^{1 / 2}$. 
For the purposes of numerical integration, it is convenient to set $w=\frac{\xi_{r}}{\frac{d \xi_{r}}{d x}}$ which transforms (19) to,

$$
\frac{d W}{d x}+p(x) W^{2}+q(x) W=1
$$

$$
W(0)=W(1)=0
$$

where,

$$
p(x)=\frac{1}{x^{2}}-2 \frac{d f / d x}{x f}+k^{2}-\frac{D_{s} x^{-2}}{4 f^{2}}
$$

and,

$$
q(x)=-\left(2 \frac{d f / d x}{f}+\frac{1}{x}\right)
$$

From (22) and the asymptotic expressions $\mathrm{f} \approx \tau, \frac{\mathrm{df}}{\mathrm{dx}} \approx 1$, we find that the criterid for entering the asymptotic regime governed by $(20)$ is that

$$
M=\left|\frac{8 \tau}{x_{0}^{2} D_{s} \bar{\Omega}^{2}}\right| \ll 1 \text {. }
$$

In this formula, the value of $\tau$ such that $M=.01$ may be taken as a measure of the thickness of the boundary layer, i.e., 


$$
T_{\text {b.l. }}=.01 \frac{x_{0}^{2} D_{s} \bar{\Omega}^{2}}{8}
$$

We note that according to (23) the boundary layer thickens as the plasma rotates faster.

\section{MÄTCHING INNER AND OUTER SOLUTIONS}

In order to match the outer solutions, the two exponentially growing solutions to the inner equations must be set equal to zero, leaving the two algebraic solutions for matching.

The condition that the algebraic inner solutions match the outer ideal MHD solutions can be exprcsscd as:

$$
\lim _{\tau \rightarrow 0} \xi_{\Gamma}(\tau)=\lim _{x \rightarrow \infty} \xi_{(x)}^{\text {inner }}
$$

From (18) and (19) it is clear that the inner solutions can be matched asymptotically to the outer solutions in the sense of (24).

We will be investigating a class of resistive eigenmodes called tearing modes; that are sensitive to the matching conditions between the inner and outer regions. We subdivide the plasma radius into three regions. Region $I$ is the outer region surrounding the magnetic axis. Generally, when treating boundary layers, the boundary layer is called the inner region, and the rest is called the outer region. Here, however, we have two outer regions, one of winich is in some 
sense inside the boundary layer. We can just call it region I. Region II is then the inner, resistive boundary layer, and region III the other outer region.

From Equation (21) we have seen that the solution in Region I near the boundary layer approaches the form:

$$
\zeta_{I, I}=A_{I}|i|^{h_{1}}+B_{I}|\cdot|^{-1=h}
$$

Likewise in Region III,

$$
\dot{\xi}_{I, I I I}=A_{I I I}|\tau|^{h}+B_{I I I}|\tau|^{-1-h}
$$

Similarly in Region II,

$$
\xi=\left\{\begin{array}{l}
A_{I I, L}|x|^{h}+B_{I I, L}|x|^{-1-h} \text { as } x,-\infty \\
A_{I I, R}|x|^{h}+B_{I I, R}|x|^{-1-h} \text { as } x \rightarrow \infty
\end{array}\right.
$$

The matching condition, (24) can be expressed as,

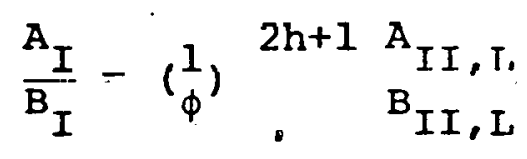

and,

$$
\frac{\mathrm{A}_{I I I}}{\mathrm{~B}_{I I I}}=\left(\frac{1}{\phi}\right)^{2 \mathrm{~h}+1} \frac{\mathrm{A}_{I I, R}}{\mathrm{~B}_{I I, R}}
$$


for $2 h+1=\left(1-\Omega^{2}\left(r_{0} D_{S}-P\right)\right)^{1 / 2}>0$ and where $\phi$ is the small scale factor.

We expect for most cases that $\frac{A_{I}}{B_{I}}$ and $\frac{{ }^{A} \text { III will be }}{B_{I I I}}$

finite numbers. Thus to obtain matching, the growth rate must be adjusted in the resistive layer so that $A_{I I, L} \approx 0$. The exceptional case occurs, when $B_{I}$ vanishes. According to Newcomb's stability criterion (NEWCOMB, 1960) this is the condition for marginal ideal stability. It is not surprising that a system that is close to being unstable in the ideal theory will have a strongly modified resistive instability:

These matching constants can be calculated numerically from the outer layer equations as the limit

$$
\begin{aligned}
\frac{A_{I}}{B_{I}} \cdot \frac{A_{I I I}}{B_{I I I}} & =\lim _{x \rightarrow \mp \infty}\left(\mp x^{-4 h-2} \frac{\frac{d}{d x}\left(x^{1+h} \xi\right)}{\frac{d}{d x}\left(x^{-h} \xi\right)}\right) \\
& =\lim _{x \rightarrow \mp \infty}\left(\mp x^{4 h-2} \frac{\frac{d}{d x}\left(x^{h} \psi\right)}{\frac{d}{d x}\left(x^{-1-h} \psi\right)}\right)
\end{aligned}
$$


In terms of $W,(29)$ can be rewritten,

$$
\begin{gathered}
\frac{A_{I I I}}{B_{I I I}}=\lim _{\tau \rightarrow 0}-|\tau|^{-2 h-1}\left[\frac{h+1+\frac{\tau}{W}}{-h+\frac{\tau}{W}}\right] \\
\therefore \\
\frac{\Lambda_{I}}{B_{I}}=\lim _{\tau \rightarrow 0}-|\tau|^{2 h-1},\left[\frac{h+1-\frac{\tau}{W}}{-h}-\frac{\tau}{W}\right]
\end{gathered}
$$

The quantity matched to the inner layer is,

$$
\Delta^{\prime}=\frac{{ }^{A_{I I I}}}{{ }^{B_{I T I}}}-\frac{{ }^{A}}{B_{I}} .
$$

Our objective in this report is to gain some information about the eigenvalue problem by purely analytic means. To do this, we consider the growth rate, 5 , ard $h=0$. It will prove convenicnt to match the $r$-component of the perturbed magnetic field at the edge of the resistive layer rather than the plasma displacements, in which case the asymptotic solutions are

$$
\begin{aligned}
q_{r, I} & =B_{I}+A_{I} \tau, \text { as } \tau+0 \\
q_{r, I I I} & =B_{I I I}+A_{I I I} \tau \text {, as } \tau+0 \text {. }
\end{aligned}
$$


and,

$\psi=\left\{\begin{array}{cc}B_{I I, L}+A_{I I, L} x & \text { as } x \rightarrow-\infty \\ B_{I I, R}+A_{I I, R} x & \text { as } x \rightarrow \infty\end{array}\right.$

As in (27) and (28) the matching conditions become,

$$
\frac{1}{\phi} \frac{A_{I I, L}}{B_{I I, L}}, \frac{1}{\phi} \frac{A_{I I, R}}{B_{I I, R}}=\dot{O}(1)
$$

We have seen that the behavior of the exponential solutions of the RIE are

$$
\xi \sim \exp \left\{ \pm \frac{k x^{2}}{2(n S)^{1 / 2}}\right\} \text { for } k \text { constant }
$$

as $x \rightarrow \pm \infty$. Thus as $S$ becomes smail, we must consider the length scale to become small as $s^{1 / 4}$, otherwise the exponential solutions would grow infinitely rapidly making the equations badly posed: That is, there is an inner layer of significant behavior that is even thinner than the resistive skin depth by another factor of $\mathrm{s}^{1 / 4}$.

We find the interesting behavior within this new scaling by expanding the parameters and variables of equations (11) - (13) in powers of $\mathrm{S}$ as follows: 


$$
\begin{gathered}
\xi=\xi_{0}+\ldots \\
\psi=s^{1 / 4} \psi_{0}+s^{7 / 4} \psi_{1}+\ldots \\
\zeta=s^{3 / 2} \zeta_{0}+\ldots \\
x=s^{1 / 4} \bar{x}, \quad \varepsilon=\frac{1}{\varepsilon^{1 / 2} \bar{\varepsilon}, \quad \delta=s^{3 / 4} \bar{\Omega}}
\end{gathered}
$$

Equations (11) - (13) reduce in the zero th ordes to:

$$
n \frac{d^{2} \psi_{0}}{d x^{2}}=0
$$

$$
\begin{gathered}
\frac{d^{2} \xi_{0}}{d \bar{x}^{2}}=\left(\frac{\bar{x}^{2}}{n}-\bar{n}^{2}\left[\frac{r_{0} D_{S}}{4}-1-F\right]\right) \xi_{0}+\left(\bar{\Omega} \bar{x}-\frac{\bar{\varepsilon}}{n} x^{2}\right) \zeta_{0}-\frac{\bar{x}}{11} \psi_{0} \\
\frac{d^{2} \zeta_{0}}{d x^{2}}=\bar{x}^{2}\left[1+\frac{\bar{\varepsilon}^{2}}{n}\right] \zeta_{0}+\left(\bar{x} \bar{\Omega}[1+P]-\frac{\dot{\varepsilon} \bar{x}^{2}}{n}\right) \xi_{0}+\left(\bar{\varepsilon} \frac{\bar{x}}{n}-P \bar{\Omega}\right) \psi_{0}
\end{gathered}
$$

In the first order:

$$
n \frac{d^{2} \psi_{1}}{d \bar{x}^{2}}=-\bar{x} \xi_{0}+\bar{\varepsilon} \bar{x} \zeta_{0}+\psi_{0}
$$

Thus

$$
\psi_{0}=B_{I I}^{(0)}+A_{I I}^{(0)} \bar{x}
$$



But since $A_{I I} \approx 0$ for matching, we are justified in setting $A_{I I}=0$ in the zeroth order and accounting for the $x$ dependence in the next order. In fact, from (31), and in view of (14), and (15), we have

$$
\frac{d^{2} \psi_{1}}{d \bar{x}^{-2}}=0 \quad \text { as, } \quad x \rightarrow \pm \infty
$$

which has solutions,

$$
\psi_{1}=\left\{\begin{array}{cc}
(1) & \bar{x} \text { as } \bar{x}+-\infty \\
A_{I I, L} & \\
(I) & \bar{x} \text { as } \bar{x} \rightarrow \infty
\end{array}\right.
$$

where the constant solution has been incorporated in the zeroth order solution. Thus the total $\psi$ solution is,

$$
\psi(\bar{x})=s^{7 / 4} A_{I I}^{(I)} \cdot \bar{x}+s^{1 / 4} B_{I I}^{(0)}
$$

And since,

$$
\frac{d \psi}{d x} \quad(x)=\frac{1}{s^{1 / 4}} \frac{d \psi}{d \bar{x}}(\bar{x})
$$

making use of $(25),(26)$ and (31), we arrive at the dispersion 'relation,

$$
\frac{1}{\phi} \Delta=\Delta^{\prime}
$$


where,

$$
\Delta^{\prime}=\frac{A_{I I I}}{B_{I I I}}-\frac{A_{I}}{B_{I}}
$$

and,

$\frac{1}{\phi} \Delta=\lim _{x \rightarrow \infty} \frac{\frac{d \psi}{d x}(x)-\frac{d \psi}{d x}(x-)}{\phi \beta s^{1 / 4}}=\frac{s^{5 / 4}}{\beta \phi} \lim _{x \rightarrow \infty} \int_{-x}^{-x}\left[-\bar{x} \xi_{n}+\bar{\varepsilon} \bar{x} \zeta_{0}\right.$.

$$
+B] d \bar{x}
$$

where we have set $B \equiv B_{I I}^{(0)} \equiv \psi_{0} \cdot$

Thus the condition that $\Delta^{\prime}=.0$ (1) requires that

$$
\phi=s^{5 / 4}
$$


6. ANALYSIS OF TEARING MODE EQUATIONS

6.1 The Scaled equations

From the original scalings of section 5 , and equation (34), the tearing mode scalings can be rewritten in terms of the scale factor $\theta$ as

$$
\begin{aligned}
& \eta=\theta^{5} \bar{\eta}, \gamma=\theta^{3} Q, \frac{d \Omega}{d r}=\theta \frac{d \Lambda}{d r} \\
& \tau=\theta^{2} \bar{x}, \varepsilon=\theta \bar{\varepsilon}, \Omega=\theta \Lambda \\
& q_{r}=\theta^{2} \psi_{0}+\theta^{4} \psi_{1}+\ldots \\
& \xi_{r}=\xi+\ldots \\
& q_{\theta}=\theta^{2}{ }_{5}+\ldots \\
& \psi_{0}=\beta
\end{aligned}
$$

and letting $\mathrm{P}=\frac{{ }_{0}}{2 \Lambda} \frac{\mathrm{d} \Lambda}{\mathrm{dr}} \quad$ and $\mathrm{q}=\frac{\varepsilon \mathrm{D}_{\mathrm{S}}}{\Lambda}$.

The following tearing mode equations result from scaling the basic equations (11) - (13) according to (36), omitting superscripts and considering $\left|P-\frac{r_{0}{ }^{D} S}{4}\right| \ll I$

$\frac{d^{2} \xi_{0}}{d x^{2}}=\left(\frac{\Lambda^{2}}{Q^{2}}\left[1-\frac{r_{0} D_{S}}{4}+P\right]+\frac{x^{2}}{Q n}\right) \xi_{0}+\left(\frac{\Lambda x}{Q^{3}}-\frac{\varepsilon x^{2}}{Q^{2}}\right) \xi_{0}$

$$
+\left(\frac{{ }^{\alpha}-\frac{1}{r_{0}}}{Q^{2}}-\frac{x}{n Q}\right) B
$$




$$
\begin{aligned}
\frac{d^{2} \zeta_{0}}{d x^{2}}=\left(\frac{x \Lambda}{n}[1+p+q]-\frac{\varepsilon x^{2}}{n^{2}} Q\right) \xi_{0}+\left(\frac{x^{2}}{Q}+\frac{\varepsilon^{2} x^{2}}{n^{2}}\right) \zeta_{0} \\
+\left(-\frac{\varepsilon}{n}\left[\alpha_{1}-\frac{1}{r_{0}}\right]-\frac{\Lambda}{n}[p+q]+\frac{\varepsilon x Q}{n^{2}}\right) B \\
\quad \frac{d^{2} \Psi_{1}}{d x^{2}}=-\frac{x Q}{n} \xi_{0}+\frac{\varepsilon x}{n} \zeta_{0}+\frac{Q}{n} B
\end{aligned}
$$

where the superscripts have been omitted from the scaled variables.

\subsection{Asymptotics}

We study the asymptotics of system (37) - (39) by expanding the independent variables as foilows:

$$
\begin{aligned}
& \xi=\frac{\xi_{1}}{x}+\frac{\xi_{2}}{x^{2}}+\ldots \\
& \zeta=\frac{\zeta}{x}+\frac{\zeta_{2}}{x^{2}}+\ldots
\end{aligned}
$$

Replacing these expansions in (37) - (39), we obtain the result that

$$
\left.\begin{array}{c}
\zeta_{1}=0, \xi_{1}=\beta, \\
\zeta_{2}=-\beta Q \Lambda, \xi_{2}=\left[\frac{\eta}{Q}\left(\frac{1}{r_{0}}-\alpha_{1}\right)-\varepsilon \Lambda\right] \beta
\end{array}\right\}
$$

and,

$\psi_{1}=\beta\left[1+\frac{\Lambda^{2} r_{0} D_{s}}{4} \ln x+\ldots\right]+\left[c_{1}+B\left(\alpha_{1}-\frac{1}{r_{0}}\right) \ln x\right] x+\ldots$

where $B$ and $c_{1}$ are chosen to match the outer solution. 
$-31-$

6.3 The dispersion relation

$$
\text { In order to study system (37) - (39) analytically }
$$

we are motivated by equations (42) to make the following change of variables:

$$
\begin{aligned}
& z=\frac{x}{\left(Q_{n}\right)^{1 / 4}} \\
& \zeta=\frac{Q^{1 / 2} \Lambda B}{n^{1 / 2} \bar{\zeta}} \\
& \xi=\frac{B}{\left(Q_{n}\right)^{1 / 4} \bar{\xi}}
\end{aligned}
$$

We also define the following parameters,

$$
\begin{aligned}
& \qquad r^{2}=\frac{\varepsilon^{2} Q}{n}, \quad \Lambda=\frac{Q^{3 / 4}}{n^{1 / 4}} \bar{\Lambda} \\
& \text { and } \bar{q}=p+q
\end{aligned}
$$

With these substitutions (37) - (39) can be

$\therefore \quad$ rewritten; assuming that $\left|\frac{{ }^{C} D_{S}}{4}-P\right| \ll 1$ :

$$
\begin{array}{r}
\frac{d^{2} \bar{\xi}}{d z^{2}}=\left[\bar{\Lambda}^{2}\left(1-\frac{r_{0} D_{S}}{4}\right)+z^{2}\right] \bar{\xi}+\bar{\Lambda}\left[\bar{\Lambda} z-r z^{2}\right] \bar{\zeta}+\left(\alpha_{1}\right. \\
\left.-\frac{1}{r_{0}}\right) \frac{n^{3 / 4}}{Q^{5 / 4}}-z
\end{array}
$$




$$
\begin{aligned}
& \frac{\mathrm{d}^{2} \zeta}{\mathrm{d} z^{2}}=\frac{1}{\bar{\Lambda}}\left(z \bar{\Lambda}(1+\bar{q})-r z^{2}\right) \bar{\xi}+z^{2}\left(1+r^{2}\right) \bar{\zeta} \\
& +\left(\frac{\varepsilon z}{\bar{\Lambda}}-\frac{\varepsilon \eta^{1 / 4}}{Q^{3 / 4} \bar{\Lambda}}\left(\alpha_{1}-\frac{1}{r_{0}}\right)-\bar{q} .\right. \\
& \frac{\mathrm{d}^{2} \bar{\psi}}{d \mathrm{z}^{2}}=\frac{\mathrm{Q}^{3 / 2}}{n^{1 / 2}}(1-\mathrm{z} \bar{\xi}+\mathrm{r} \bar{\Lambda} \bar{\zeta})
\end{aligned}
$$

In the text that follows we will simplity the computation by taking $\alpha_{1}=\frac{1}{r_{0}}$.

The dispersion relation (32) - (34) can also be rewritten in terms of these substitutions:

$$
\frac{I}{B}\left[\frac{d \psi}{d x}\right] \frac{Q^{5 / 4}}{n^{3 / 4}} F
$$

where,

$$
F=\lim _{z \rightarrow \infty} \int_{-z}^{z}(1-z \bar{\xi}+r \bar{\Lambda} \bar{\zeta}) d z
$$

and,

$$
\frac{1}{B}\left[\frac{Q \psi}{d x}\right]=\frac{1}{B} \lim _{x \rightarrow \infty}\left[\frac{d \psi}{d x}(x)-\frac{d y}{d x}(-x)\right]=\Delta
$$

Thus, the dispersion relation is:

$$
\Delta^{\prime}=\Delta
$$


and making use of (36) we have:

$$
\bar{\gamma}=\bar{n}^{3 / 5}\left(\frac{\Delta}{F}\right) 4 / 5
$$

where the dimensionless quantities $\bar{\gamma}$ and $\bar{\eta}$ have been used in place of the scaled variables $Q$ and $n$, and $F$ depends on the parameters in equations (44) - (46), namely, $\bar{q}, r, \bar{\Lambda}$, and $D_{s}$.

$6.4 \cdot$ A special case

Our objective here is to find a special.

class of analytic solutions to the inner layer equations (44) and (45), and use these solutions to evaluate the dispersion relation (48) explicitly.

The case we consider is characterized by,

$\frac{r_{0} D_{S}}{4} \ll 1, \quad r \ll 1, \quad P=0, \bar{q}=-1, \bar{\Lambda}=0(1)$

This corresponds to a special case of a theta pinch equilibrium with rigid body rotation and density gradient opposite in sign to the angular velocity. 
From (44) we see that only the odd part of $\bar{\xi}$ and $\bar{\zeta}$ contribute to the dispersion relation. Furthermore, in the limit of small $Q$, the second term in (44) can be treated as a perturbation, thus only the odd part of $\bar{\xi}$ must be determined in order to derive the dispersion relation: But, referring to (43) and (44), and using. the result of the asymptotics summarizcd in (14) and (15), all terms involving $r$ may be treated as pertubations and neglected to leading order. Thus $(44)-(46)$ reduces to:

$$
\begin{aligned}
& \frac{\mathrm{d}^{2} \bar{\xi}}{\mathrm{d} \mathrm{z}^{2}}-\mathrm{z}^{2} \bar{\xi}-\bar{\Lambda}_{0}^{2} \bar{\xi}=z \bar{\Lambda}_{0}^{2} \bar{\zeta}-2 \\
& \frac{\mathrm{d}^{2} \bar{\zeta}}{\mathrm{d} \mathrm{z}^{2}}-z^{2} \bar{\zeta}=1
\end{aligned}
$$

$$
\frac{1}{\beta}\left[\frac{d \|^{\prime}}{d x}\right]=\frac{1}{(n Q)^{1 / 4}} \int_{-\infty}^{\infty} \frac{Q^{3 / 2}}{n^{1 / 2}}(1-z \bar{\xi}) d z
$$

where we denote the value of $\bar{\Lambda}$ corresponoing to the special case $q=-1$ by $\bar{n}_{0}$. We let,

$$
\begin{aligned}
& \bar{\zeta}=\varepsilon^{-z^{2} / 2}\left[b_{2 k} H_{2 k}(z)\right. \\
& \bar{\xi}=\varepsilon^{-z^{2} / 2}\left[a_{2 k+1} H_{2 k+1}(z),\right.
\end{aligned}
$$

where $\mathrm{H}_{2 \mathrm{k}}, \mathrm{H}_{2 \mathrm{k}+\mathrm{I}}$ are Hermite polynomials. 
and make use of the identities,

$$
\left.\begin{array}{l}
1=\sqrt{2} \varepsilon^{-z^{2} / 2} \frac{H_{2 k}(z)}{2^{2 k_{\Gamma}(k+1)}} \\
z=\sqrt{2} \varepsilon^{-z^{2} / 2} \frac{H_{2 k+1}(z)}{2^{2 k_{\Gamma}(k+1)}}
\end{array}\right\}
$$

Solving for $\bar{\xi}$ we find,

$$
a_{2 k+1}=\frac{1}{2^{2 k+\frac{1}{2} \Gamma(1+1)}} \frac{\left[2+\bar{\Lambda}_{0}\left(\frac{1}{4 k+1}+\frac{1}{4 k+5}\right)\right]}{\left(4 k+3+\bar{\Lambda}_{0}^{2}\right.}
$$

Replacing $\bar{\xi}$ in (51), and using the orthogonality

relations for Hermite polynomials yields,

$$
\Delta=\frac{\pi \bar{r}^{-5 / 4}}{2 \bar{n}^{-3 / 4}} F\left(\bar{\Lambda}_{0}\right)
$$

where,

$$
F\left(\bar{\Lambda}_{0}\right)=-4\left(1+\bar{\Lambda}_{0}^{2}\right) \frac{\Gamma\left(\frac{3+\bar{\Lambda}_{o}^{2}}{4}\right)}{\Gamma\left(\frac{5+\bar{\Lambda}_{0}^{2}}{4}\right)}+2 \bar{\Lambda}_{\circ}^{2} \frac{\Gamma\left(\frac{1}{4}\right)}{\Gamma\left(\frac{3}{4}\right)} \text {. }
$$

Growth rates can be obtained by solving the dispersion relation, $\Delta^{\prime}=\Delta$, for the growth rate as given by (49) where the quantity $\Delta^{\prime}$ is determined by integrating the ideal equations of motion forward from $r=0$ and backward from $r=r_{w}\left(r_{w}=\right.$ wall position $)$ and then forming the logarithmic derivative as indicated in Eq. (30a). From (49) we notice 
that the stability criterion is $\Delta^{\prime}>0$, that is, for positive values of $\Delta^{\prime}$ the plasma is unstable and positive growth rates can be calculated. For $\Delta^{\prime} \leq 0^{\circ}$, which depends only on equilibbrium quantities outside the resistive layer, the plasma is stable. We note that, in general, the dispersion relation will have to be solved iteratively since from (43), $\bar{\Lambda}_{0}$ depends on the growth rate.

6.5 valldatiun of the analyeis

The ultimate goal in this work is to compute the stability (growth rates and stability boundaries) of realistic plasma configurations to the tearing modes considered here. Before proceeding, it is improtant to validate the analysis with a specific example. Let us consider the special case of the last section. We will show by a numerical integration of. the governing equations in the resistive layer that i) the asymptotics are correct and ii) the analytic solutions given by (53) and (54) are correct. We use a technique (FISCHER, 1969) for solving Eqs. (50) - (52) which converts the boundary value problem into an initial value problem. This technique has been used successfully elsewhere (JARDIN) in a number of resistive boundary layer calculations. We have chosen the special case $q=-1, \bar{\Lambda}^{2}=0.75$ and have numerically integrated the resistive layer equations for $\bar{\xi}$ and $\bar{\zeta}$ as a function of $z$ for $0<z<4$. Shown in figure la is a curve. of $\bar{\xi}$ vs. $z$; the crosses denote the solution as given by the analytic expression (54) and the circles denote the results 
$-37-$

from the numerical integration of Equation (50) and (51). Figure lb presents a similar comparison between analytic and numerical solutions for $\bar{\zeta} \cdot v s . z$. The asymptotic expansions for $\bar{\xi}$ and. $\bar{\zeta}$. for large $z$, ie. $\bar{\xi} \sim 1 / z$ and $\bar{\zeta} \sim 1 / z^{2}$, are borne out by both the numerical and analytical solutions. Further, comparison of the soldtrons. for the entire $z$ range indicates excellent agreemont. We believe therefore that the numerical technique for solving the RLE equations is satisfactory. 


\section{GENERAL RESULTS}

7.1 Effect of Hall current on the growth rate

From equation (49) we see that the effect of Hall current on the growth rate is entirely contained within the quantity $F\left(\bar{\Lambda}, q, r, D_{S}\right)$. The value of $F$ is governed, for the most part, by the parameters of the inner layer, namely, $\varepsilon, \eta$ and the local values of the angular velocity, $\Omega$, and the density gradient. $n_{s}$. Thus the quantity $F$ may be considered as a criteria of merit which gives us a means of evaluating the effect of Hall current on the growth rate of the tearing mode as compared to growth rates in the absence of Hall current. For example in the absence of Hall current and the case of rigid body rotation, $\cdot \varepsilon=\bar{q}=0$ and $F=F\left(\bar{\Lambda}, 0,0, D_{s}\right)$. Thus larger values of $F$ in the presenre of Hall current compared to no Hall current would according to (49) tend to deprcos the value of the growth rate whereas smaller values of $F$ would tend to have the reverse effect of enhancing the growth of the tearing mode.

It is important, therefore, to examine the criteria of merit, F, more closely. We will demonstrate later that for realistic values of the parameters; $F$ depends only on $\bar{\Lambda}^{2}$ and $\bar{q}$. In other words $F=F\left(\bar{\Lambda}^{2}, \bar{q}\right)$, and the parameter $r$ along with the explicit dependence of $F$ on $n_{s}$ through the term $\frac{r_{0}^{D} s}{4}$ in equation (44) may be considered as small perturbations. This simplifies the analysis of the -inner layer considerably and enables us to illustrate the dependence of 
$F$ on $\bar{\Lambda}^{2}$ and $\bar{q}$ by Figure 2 . If we consider rigid body rotations, the curve $\bar{q}=0$ corresponds to the case of no Hall current. From this figure it is evident that values of $\vec{q}>0$ result in enhanced stability to tearing modes whereas values of $\bar{q}<0$ result greater instability. For the case of rigid body rotation, from (43), $\bar{q}=\frac{\varepsilon D_{s}}{\bar{\Omega}}$. Thus rotation of the plasma in one direction relative to the value of the density gradient at the neutral point, enhances the stability to tearing modes while rotation in the opposite direction results in greater instability. This result was observed by. KALEK (1972).

Figure 2 also shows that the effect of rotation on $F$, and therefore the growth rates, is comparatively small for the case of no Ball current due to the relative constancy of the curve $q=0=\varepsilon$ compared to curves with larger values of $q$.

It should be noted that in the case of no Hall current but a sheared velocity profile, $\bar{q}=\frac{r_{0}}{2 \bar{\Omega}} \frac{d \bar{s}}{d r}$, Figure 2 shows that sheared velocity can also effect the stability of tearing modes. This point will not be pursued in this paper. Hereafter, we will specialize our analysis to rigid body rotations. 
7.2 Effect of the equilibrium and position of the outer wall on the tearing mode

The equilibrium determines the parameters of the inner layer and. thus influences the tearing modes through the criteria of merit, F. The equilibrium also affects the tearing modes through the outer equations via the parameter $\Delta^{\prime}$. Furthermore, since by equation (49), the value of $\Delta^{\prime}$ is extremely sensitive to the position of the outer wall surrounding the vacuum region, we see that growth rates and thus values of the inner parameter, $\bar{\Lambda}^{2}$ defined by (43), are very sensitive to the outer wall position. Thus values of $\vec{\Lambda}^{2}$ determine not only the effect of rotation but also of the position of the wall on the value of the criteria of merit., F.

We now show how the Hall current, rigid body rotation, and wall position effect the growth rates of tearing modes for a family of rigid rotor, Theta pinch equilibria. To be exact, we will determine an overestimate of the ratio of growth rate with Hall current to growth rate with no Hall current, and show how these ratios are affected by angular velocity for fixed positions of the outer wall. '. The next section is devoted to describing the equilibria. 


\section{RESULTS FOR A SPECIFIC FAMILY OF EQUILIBRIA}

In this section we apply the theory to a class of reversed field, isothermal, rigid-rotor Theta pinch MHD equilibria. We assume that all the mass and rotational energy is carried by the ions and that the family of equilibria is parametized by the angular velocity, $\Omega$, and characterized by a constant number of particles, $\mathrm{N}_{\mathrm{L}} \cdot$ The equilibria are one dimensional, and although their domain of definition is from the plasma center line, $I=0$, to $I=\infty$, the density is effectively zero for $r \geq a$; where a is taken to be the plasma radius. Furthermore all equilibrium quantities are constant from the plasma. edge to the wall of a vacuum region, $r_{w^{\prime}}$ surrounding the plasma.

8.1 The equilibrium equations

The equilibrium is characterized by the force balance,

$$
\frac{d}{d r}\left(p+\frac{i}{2 \mu_{0}} B^{2}\right)=\Omega^{2} r p
$$

and the cquation of statc,

$$
p=c p ; c=\frac{K T}{m_{i}}
$$

where $k$ is Boltzmann's constant, $m_{i}$ is the mass of an ion, and $T$ is the plasma ion temperature. 
The number of particles per unit plasma column length (ions), $\mathrm{N}_{L^{\prime}}$ is determined by,

$$
N_{L}=\frac{2 \pi}{m_{i}} \int_{0}^{a} \rho(r) r d r
$$

and expressed in terms of a characteristic radius, $r_{\ell}$, as

$$
N_{L}=\pi_{i} \rho(0) \mathbf{F}_{l}^{2}
$$

We consider the following ansatz,

$$
\rho(x)=\rho(0) \operatorname{sech}^{2}\left(\left(\frac{x}{x_{p}}\right)^{2}-\xi_{0}\right)
$$

and,

$$
\left.B_{z}=B_{\infty}\left(\delta+(1-\hat{u}) \tanh \left(\frac{x}{x_{p}}\right)^{2}-\xi_{0}\right)\right)
$$

where, all lengths are normalized to the plasma radius, i.e., $x=\frac{r}{a}$.

Replacing (58) and (59) in (55) and (56) and charaatcrizing the ratio of the thermal to the magnetic energy by,

$$
B=2 c \cdot \frac{\rho(0) \mu_{0}}{B_{\infty}^{2}},
$$

and the ratio of the rotational to the thermal energy by

$$
\Omega^{* 2}=\frac{\Omega^{2} a^{2}}{2 c} B
$$


yields,

$$
\begin{gathered}
\Omega^{\star 2}=\frac{2 \delta(1-\delta)}{x_{p}^{2}} \operatorname{sech}^{2} \xi_{0} \\
B=(1-\delta)^{2} \operatorname{sech}^{2} \xi_{0} \\
x_{p}^{2}=x_{l}^{2} \frac{\operatorname{sech}^{2} \xi_{0}}{1+\tanh \xi_{0}}
\end{gathered}
$$

The equilibrium is therefore entirely specified once the physically meaningful variables, $B_{\infty}, \rho(0), T, N_{L}$ and $\Omega$ are specified. However, for our purposes it is more convenient to specify, $B_{\infty}, N_{L}, \xi_{0}, \delta$, and $x_{\ell}$.

In terms of the latter set of parameters, the plasma temperature, rigid body rotion and the neutral point, $x_{0}$ ' of the plasma are given by,

$$
\begin{gathered}
\Omega^{2}=\frac{2 \pi \delta(1-\delta)\left(1+\tanh \xi_{0}\right)}{m_{i} \mu_{0} N_{L}} \\
T=\frac{m_{i} c}{K}
\end{gathered}
$$

where,

$$
c=\Omega^{2} a^{2} x_{\ell}^{2}\left(1-\tanh \xi_{0}\right)\left(\frac{1-\delta}{4 \delta}\right)
$$


and,

$$
x_{0}=x_{\ell}\left(1-\tanh \xi_{0}\right)^{1 / 2}\left(\xi_{0}+\frac{1}{2} \ln (1-2 \gamma)\right)^{1 / 2}
$$

The object of this study is to show the effect of the Hall current dominated boundary layer on the growth rates, $\gamma$, of the tearinq modes expresed by the dispersinn relation,

$$
\bar{\gamma}=\bar{n}^{3 / 5}\left(\frac{\Delta^{\prime}}{F\left(\bar{\Lambda}^{2}, q\right)}\right)^{4 / 5}
$$

where, once again, the superscripted variables are normalized with respect to the boundary layer quantities and the variables $q$ and $\bar{\Lambda}^{2}$ governing the inner equations are defined by (36a), and (43). Valucs of the mismatch, $\Delta^{*}$, are obtained hy integrating the outer equations as described in section 7 while $F\left(\bar{\Lambda}^{2}, q\right)$ is obtained by integrating the inner equations as described in section 8. . Since $\bar{\gamma}$ occurs on both sides of the equation, (61) must be solved iteratively.

The inner variables and the normalizations for (61) are computed as follows:

$$
\begin{aligned}
q & =\frac{\varepsilon \Omega}{1-\delta}\left(\xi_{0}+\frac{1}{2} \ln (1-2 \gamma)\right) \\
\bar{\Lambda}^{2} & =\frac{\bar{n}^{1 / 2} \bar{\Omega}^{2}}{\bar{\gamma}^{3 / 2}}
\end{aligned}
$$


$-45-$

$$
\begin{aligned}
& r^{2}=\frac{2 \varepsilon^{2} \delta c \mu_{0}}{n(1-2 \delta)} \\
& \bar{n}=\frac{n}{a \bar{k} \mu_{0} V} \\
& \bar{\Omega}=\frac{2 \Omega a}{v} \\
& \bar{r}=\frac{\gamma a}{\bar{k} v}
\end{aligned}
$$

where,

$$
\overline{\mathrm{k}}=2 \pi \frac{\mathrm{a}}{\mathrm{L}} .
$$

and, $(L=$ perturbation wavelength)

$$
v^{2}=\frac{4 a^{2} \pi\left(\xi_{0}+\frac{1}{2} \ln (1-2 \delta)\right)\left(1+\tanh \xi_{0}\right)(1-2 \delta)}{\mu_{0} m_{i} N_{L}}
$$

and from equation (Ila)

$$
\text { h. }=-\frac{1}{2}+\frac{1}{2}\left[1+\frac{8 \delta^{2}}{1-2 \delta}\right]^{1 / 2}
$$


8.2 Results of tearing mode analysis

We carried out the analysis of the above equilibrium for the following range of parameters:

$$
\begin{aligned}
& B_{\infty}=1, \xi_{0}=1, x_{\ell}=1, \\
& \varepsilon=1.02 \times 10^{-8} \varepsilon-\frac{M H O-K G}{\mathrm{~m}^{2} \text { weber }}, \eta=.12 \frac{\mathrm{MHO}-\mathrm{KG}}{\mathrm{m}^{4}}, \mu_{0}=4 \pi \times 10^{-7} \\
& k=1.38 \times 10^{-22} \frac{\mathrm{kg}-\mathrm{m}^{2}}{o_{k} \sec ^{2}}, m_{i}=3.2 \times 10^{-27} \mathrm{~kg} \\
& \mathrm{a}=.05 \mathrm{~m}, \frac{\mathrm{a}}{\mathrm{L}}=.05 \\
& N_{L}=1 \times 10^{16}-1 \times 10^{18} \text { (ions) }
\end{aligned}
$$

and,

$$
\delta=.025-.425
$$

where $\delta$ is the normalized angular velocity defined by $(60)$.

As we discussed in the previous sections, the effect of the resistive layer on the teariny modes is contained entirejy in the criteria of merit, F, whereas the outer regions affect the tearing modes primarily through the mismatch $\Delta^{\prime}$. Finally, there is a feedback between outer and inner regions that is particularly sensitive to the position of the outer wall, since the position of the outer wall directly affects $\Delta^{\prime}$, and indirectly affects $F$ through $\bar{\Lambda}^{2}$. As we mentioned above, $F$ depends to leading order, on the parameters $q$ and $\bar{\Lambda}^{2}$, since for the equilibria. 
being considered, the parameter $r<10^{-3}$ while $\frac{r_{0} D_{S}}{4}$, according to Table 1, varies from 0 to-.23. This range of values of $\frac{r_{0} D_{s}}{4}$ has been shown to have only a minor effect on the results.

More important, as shown in section 8 , it is basic to the tearing mode analysis that the exponent, $h$, defined by equation (21a), be real. Furthermore, the asymptotic analysis requires that $h \tilde{\sim} 0$. Equation (65) shows that real $\mathrm{h}$ is guaranteed for $\delta<.5$, while Table 1 indicates that reasonable results can be expected from the asymptotic analysis for $\delta<.2$. However, we carry out the analysis for values of $\delta$ as high as .425 and expect the results to have qualitative validity.

Exact values of the growth rate may be obtained by using the following procedure: 
1. Fix the position of the outer wall at some point at which the equilibrium has an unstable tearing mode for the above values of the equilibrium parameters and angular velocity corresponding to $\delta=.025$. As mentioned above, the criteria for such an instability is that $\Delta^{\prime}>0$ where $\Delta^{\prime}$ is computed from equation (30b).

2. At this low value of the angular velocity, the parameter $\bar{n}^{2}$ governing the inner cquatinns is offuctivcly scro. Thus the criterion of merit, $F$, that characterizes the effect of Hall current and rotation, takes a value approximately equal to its vertex value of $F=2.12$ as shown in Figure 3 . The normalized growth rate, $\bar{\gamma}$, can then be calculated from:

$$
\bar{r}=\bar{n}^{3 / 5} \cdot\left(\frac{\Delta^{\prime}}{F}\right)^{4 / 5}
$$

where $\bar{n}$ is computed from $(64)$.

3. The angular velocity specified by $\delta$ is increased by a small amount keeping the particle number, ${ }^{N_{L}}{ }^{\prime}$ fired. The outer equations are reintegrated to obtain a new value of $\Delta^{\prime}$. The value of $\mathrm{q}$ is determined from equation (62) and since $\mathrm{F}$ depends also on $\bar{\Lambda}^{2}$ which by equation is a Function of the growth rate $\bar{\gamma}$, the growth rate must be determined this time from equation (61) by iteration. This iteration is initiated by using the value obtained for $\bar{\gamma}$ from step 2 as the first guess. 
4. Step 3 is repeated for the case of no Hall current, i.e., $\varepsilon=q=0$. The values of the growth rates, are used as normalizing factors; i.e., $\gamma_{\text {normalized }}=\frac{\bar{\gamma}}{\bar{\gamma}_{\varepsilon}}=0^{\circ}$

In this way a table of normalized growth rates as a function of angular velocity for a constant wall position would be obtained for one family of equilibria parametized by $\mathrm{N}_{L}$. There is however one problem which makes the implementation of the above procedure difficult. The determination of $\Delta^{\prime}$ requires the evaluation of $(30 \mathrm{~b})$ at the edge of the boundary layer. However, as shown in Table 1, the thick-

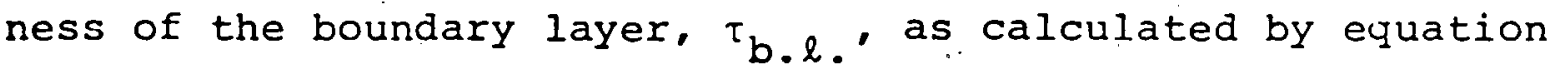
(23), varies directly with angular velocity taking on values on the order from 0 to $10^{-3}$. However; this close to the neutral point, where $\mathrm{B}_{z}\left(\mathrm{r}_{0}\right)=0$, the solutions of the outer equations are asymptotically large and the integration routines are no longer sufficiently accurate. It turns out that for some parameter ranges there exists a small annulus about the neutral point in which the plasma position is close enough to the neutral point for a computation of $\Delta^{\prime}$ to be made yet far enough away from the neutral point to enable the outer equations to be aceurately intcgrated, but this is generally not the case.

We can avoid this difficulty of integrating the outer equations if we are willing to settle for overestimates of the growth rates and incomplete information as to the 
exact position of the outer wall. We merely fix the value of $\Delta^{\prime}$ at its maximum value over the range of values on the interval of angular velocities of interest. This corresponds to. some position of the outer wall. We see from equation (61) that this value of $\Delta^{\prime}$ yields an overestimate of $\bar{\gamma}$ whereas from equation (63) for $\bar{\Lambda}^{2}$, overestimates of $\bar{\gamma}$ yield smaller values of $\bar{\Lambda}^{2}$ for the same value of $q$ thus from Fiqure 3 this results in lower valuas of $F$ and once again from equation $(61)$, overestimates of $\bar{\gamma}$. We have implemented this procedure and presented the results in Figures 3-5, where $\frac{Y}{\gamma}_{\varepsilon}=0$ is plotted against the normalized angular velocity, $\delta$, for different but unknown; outer wall positions characterized by and varying directly wilh $\Delta$ ' and for three values of the number of particles, $\mathrm{N}_{L}=10^{16}, 10^{17}, 10^{1.8}$. Actual values of the rigid hody angular velocity, $\Omega$, and ion temperature $T$ are indicated in figure 3.

Figures $3-5$, show that the inclusion of a Hall current results in increased stability to tearing modes when the plasma column rotates in one dirertion and dccrrnsed slability when the column rotates in the opposite direction. Figures 3-5 show further that, in general, both stability and instability are enhanced by rotating the column faster in either direction although above a critical value of the wall position, $\Delta^{\prime}$, the normalized growth rate, $\frac{\gamma}{\gamma_{\varepsilon}}=0$.' 
attains a maximum with respect to the normalized angular velocity, $\delta$, for rotations in one direction and a minimum for rotations in the opposite direction.

Figures $3-5$ also show that the growth rates are very sensitive to the wali position, $\Delta^{\prime}$ ', and that the plasma column becomes more stable to tearing modes for outer wall positions nearer to the plasma vacuum interface, i.e., for smaller values of $\Delta^{\prime}$. Furthermore, for sufficiently small values of $\Delta^{\prime}$, at a critical value of the normalized, angular velocity, $\delta$, the tearing mode instability is quenched, i.e., $\frac{Y}{\varepsilon}_{\varepsilon}=0$

Figures 3-5. also show that for a given wall position, $\Delta^{\prime}$, the stabilizing effect is more pronounced at lower values of the number of particles, $\mathrm{N}_{\mathrm{L}}$, and higher ion temperatures, $T$. 


\section{CONCLUSIONS}

Analysis of the linearized MHD equations of motion for a rotating field-reversed theta pinch plasma configuration has shown that the existence of Hail currents alters the dispersion realtion of unstable tearing modes in a manier which is consistent with experimentally observed behaviour. specifically the $m=0, k_{z} \neq 0$ tearing mode growth rate can be reduced for preferred rotarlonal directions. We find that overestimates of the offect of Hall current and rotation on the growth rate of tearing modes can be determined from characterisitcs of the equilibrium at the neutral point of the reversed magnetic field provided that the equilibriun is unstable to tearing modes: The effect of the tearing mocie is characterized by the criteria of merit 'F which depends, to leadins order, on on $y$ y two parameters $q$ and $\bar{\Lambda}^{2}$ When $q<0$ the Hall current always enhances the stability of the plasma system to tearing modes while values of $q$ - 0 always result in reduced stability. The parameter $\bar{\Lambda}^{2}$ is governed primarily by the position of the outer wall and the value of the angular velocity.

For a given number of particles, the stabilizing effect of the Hall current is enhanced by wall positions closer to the plasma vacuum interface. For wall. positions closer to the plasma than a critical value, the tearing mode instability is quenched at a critical value of the angular vclocity of the columu. For wall positions larger than this critical value, the growth rate attains a minimum at one value of the angular velocity for rotation in 
one direction, and attains a maximum. for another value of angular velocity for rotation in the opposite direction. For a given wall position, the stabilizing effect of the Hall current is more pronounced. for fewer total particles, $N_{L}$, and higher ion temperature, $\mathrm{T}$.

\section{Acknowledgements}

The work described in this paper was partially supported by the U.S. Department of Energy under Contract No. EY-76-C-02-3077 to New York Univorsity, and by the Office of Naval Research under Contract to New Jersey Institute of Technology, ONR-N0014-78-C-0264. The authors would like to thank S. Jardin for key discussions concerning numerical integration of the boundary layer equations and stimulating discussions with J.I. Johnson, J.M. Greene, A. Glasser, J.A. Tataronis, P. Rosenau and M. Schmidt. 


\section{BIBLIOGRAPHY}

BODIN, H.A.B., Nucl. Fusion 3, 215 (1963).

CHEN, I., RUTHERFORD, P.R., Tang, N., Phys. Rev. Letters, 39 , $460(1977)$.

COPPI, B., GREENE, J.M., JOHNSON, J.L., Nuc1. Fusion 6, (1966). EBERHAGEN, A., GROSSMANN', w., Z. Physik, 248, 130 (1971). ES'KOV, A.G., KURTMULLAYEV, R.K., MALYUTUN, A.I., PROSHLETSOV, A.P.; SEMFNOV, $V: N .$, MARYIN, $\lambda . I$, , MIRONOV, B.IN., sosunov, V.B., Conf. Plasma Phys. and Contr. Nucl. Fusion. FISCHER, C.F., USMANI, R.A., SIAM J. Numer. Anal., 6, 1 (1969). FURTH, H.P., KILLEEN, J., ROSENBLUTH, M.N., PhYS. Fluids $\underline{6}$, $459(1963)$

Innsbruck, IAEA-CN-37/U-4-1, (1978).

JARDİN, S., Privațe Communication

KALEKK, A., KÖNEN, L., NOLL, P., SIGUTA, K., WAELBROEK, F., WATANABE, K., WITULSKI, H., conf. Plasma phýs. and contr. Nucl. Fusion, Novosibirsk, Vol, II; 581 (1968).

KALEK, A., 5th Europ. Conf. Contr. Fusinn and Placma Phy3., Grenoble (1972).

LINFORD, R.K., PLȦTTS, D.A., SHERWOOD, E.G., Ibid., IAEA-CN $-37 / \mathrm{S}-1-1$

NEWCOMB, W.A., Annals of Physics, 10, 232, 267 (1960). WASOW, W., "Asmptotic Solutions to Differential Equations", Ințerscience, New York, (1965). 
$-55-$

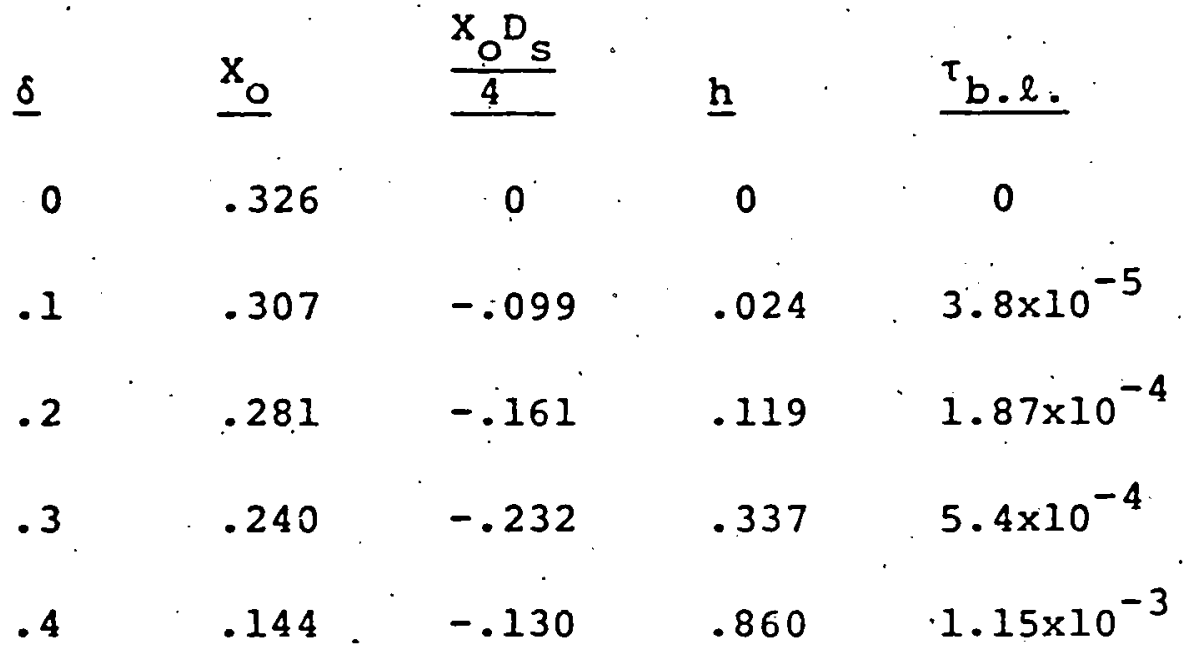

Table 1

Validity of the Analysis. 


\section{Figure Captions}

Figure 1a: Analytical solution, $\xi(z)$, the resistive layer equations for a special case (numerical solution coincides with the analytic solution within the accuracy of the graph).

Figure lb: Analytic solution, $\zeta(\mathrm{z})$, of the inner equations for a special case (numerical solution coincides wilh analytic solution within the accuracy of the graph).

Figure 2: Dependence of the criteria of merit, $F$, on the parameters $\bar{\Lambda}^{2}$ and $q$ governing the resistive layer.

Figure 3: Results of the analysis showing the effect of the normalized growth rate $\frac{\gamma}{\gamma_{\varepsilon=0}}$ on the normalized angular velocity of the plasma $\delta$; and a measure of the outer wall position, $\Delta$ ', for partirle number, $\mathrm{N}_{\mathrm{L}}-10^{16}$. Thc actual angulal veloctty of the plasma column is noted on lle abscissa.

Figure 4: Results of the analysis showing the effect of the normalizcd growth iale $\frac{Y}{\gamma}$. on the normalized angular - velocity of the plastla 8 , and a measure of the outer wall position, $\Delta^{\prime}$, for particle number, $\mathrm{N}_{\mathrm{T}}=10^{17}$. The actual angular velocity of the plasma column is noted on the abscissa. 
Figure 5: Results of the analysis showing the effect of the normalized growth rate $\frac{Y}{\gamma}_{\varepsilon=0}$ on the normalized angular velocity of the plasma $\delta$, and a measure of the outer wall position, $\Delta^{\prime}$, for particle number, $\mathrm{N}_{\mathrm{L}}=10^{18}$. The actual angular velocity of the plasma column is noted on the abscissa. 


$$
\sqrt{2}
$$


Figure $1 b$

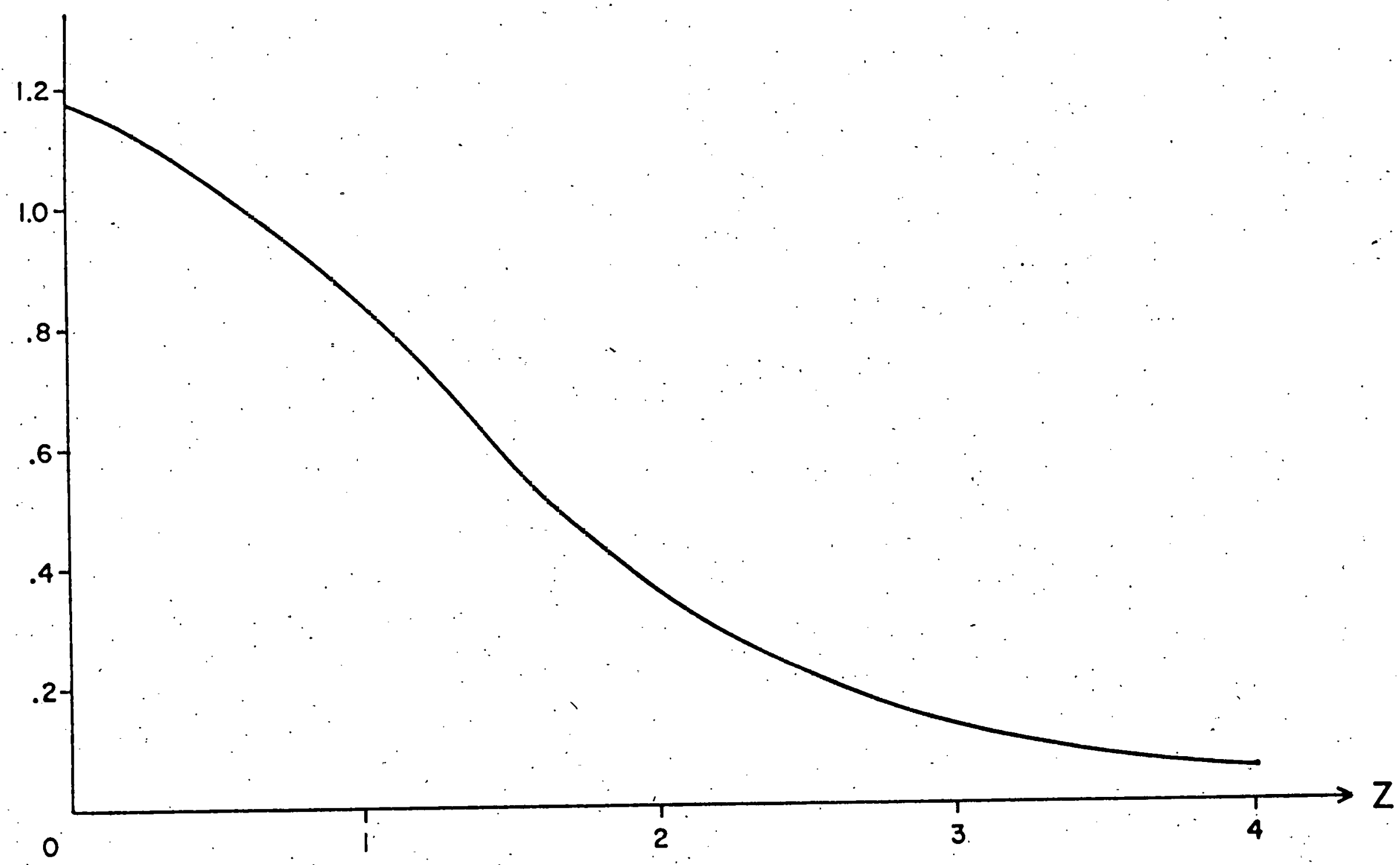




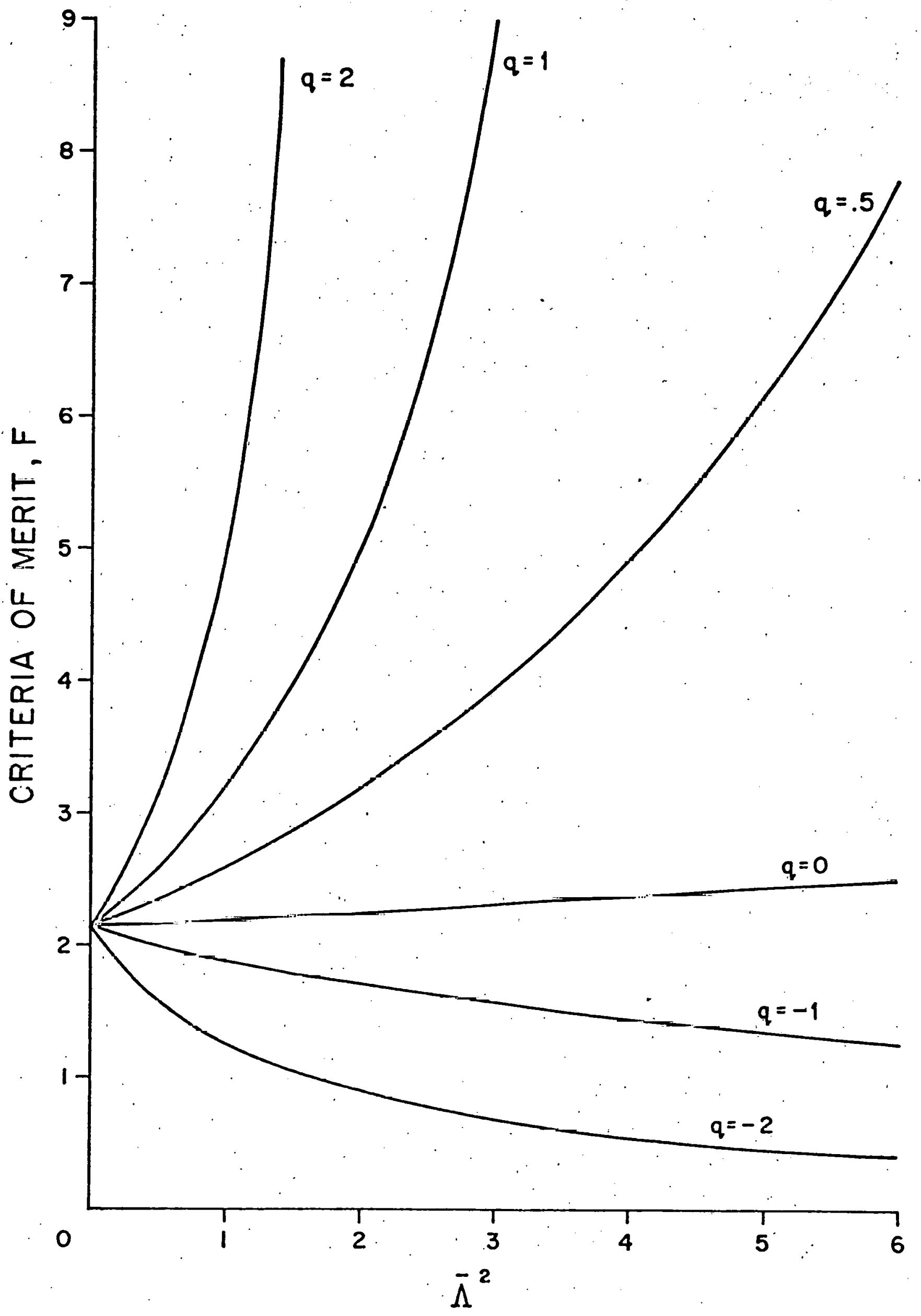




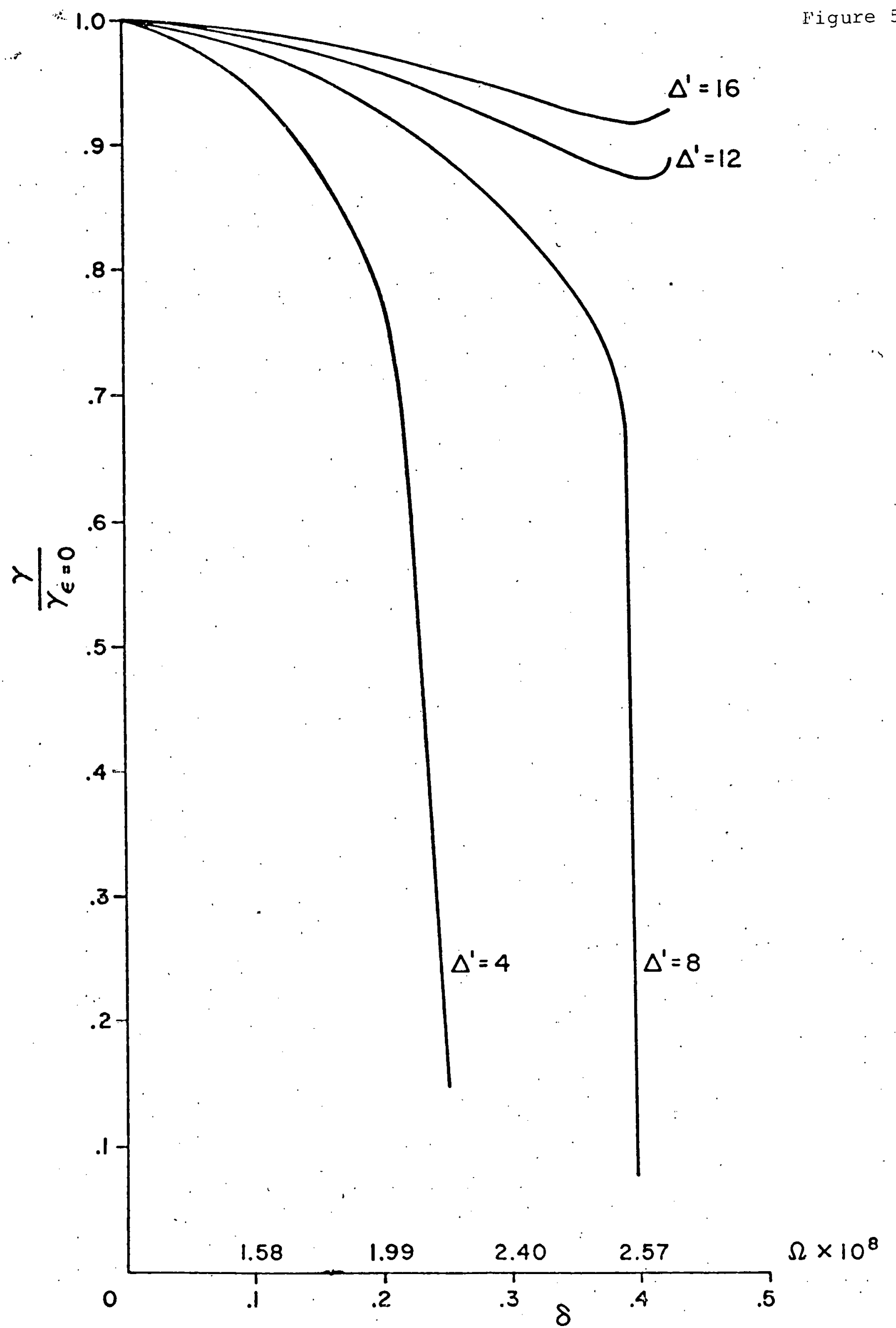

